\title{
Assessing the Structure-property Relationship in Enamel at the Nanoscale Using 4D-STEM
}

\author{
Paul Smeets ${ }^{1}$, Roberto dos Reis ${ }^{1}$, Anahita Pakzad ${ }^{2}$ and Derk Joester ${ }^{1}$ \\ ${ }^{1}$ Northwestern University, Evanston, Illinois, United States, ${ }^{2}$ Gatan Inc, Pleasanton, California, United \\ States
}

Dental enamel is a major component of teeth, which has evolved to bear substantial mastication forces, withstand wear, and resist mechanical fatigue over decades of usage. Due to its high mineral content ( 96 wt $\%)$ and its complex 3D hierarchical structure across length scales, enamel is the hardest tissue in our body ( 2-6 GPa). Despite this remarkable hardness, loss and/or functional impairment of the enamel structure with time happens nevertheless, which has a remarkable impact on health and quality of life, besides the associated significant costs to society.

The fundamental building blocks of enamel are electron beam-sensitive nanoscale crystallites of hydroxylapatite $\left(\mathrm{OHAp}\right.$; $\left.\mathrm{Ca} 5\left(\mathrm{PO}_{4}\right)_{3}(\mathrm{OH})\right)$, known to contain small quantities of other ions, such as $\mathrm{Mg}^{2+}$, $\mathrm{Na}^{+}, \mathrm{CO}_{3}{ }^{2-}$, and $\mathrm{F}^{-}$, which can substitute in the OHAp lattice [1]. Our previous work demonstrated using atomic-scale quantitative imaging (atom probe tomography; APT) and correlative spectroscopies (cryoSTEM-EELS and XANES) that these crystallites are comprised of a core enriched mainly in magnesium and other adjacent substitutional defects $\left(\mathrm{Na}^{+}, \mathrm{F}^{-}, \mathrm{CO}_{3}{ }^{2-}\right)$; this core is surrounded by a shell with lower concentration of such defects [2]. A mechanical model based on DFT calculations and X-ray diffraction data predicted that significant residual stresses arise because of such chemical gradients [2]. Although these results were obtained for several crystallites, the investigated field of view (FOV) in cryoSTEM imaging (as well as in APT reconstructed volumes) at atomic resolution was rather limited. Additionally, the mechanical strain model has not yet been verified experimentally.

In order to obtain large area low-dose CBED patterns of enamel crystallites, we used a K3 IS direct detector (Gatan, Inc). While our previous nano- and atomic scale images were collected on a conventional annular detector using FIB-prepared samples cooled to cryogenic temperatures (maximum dose of $\sim 6.5 \times$ $10^{3} \mathrm{e}^{-} \AA^{-2}$ before the onset of observed beam damage), the K3 IS allowed us to rapidly collect latticeresolution CBED data of FIB-prepared samples at room temperature (600 fps; maximum dose of $\sim 10^{3}$ $\mathrm{e}^{-} \AA^{-2}$ ). We acquired 4D-STEM datasets across a large FOV ( $>10^{4} \mathrm{~nm}^{2}$; several times larger than acquired cryoSTEM images), which allowed us to visualize many enamel crystallites. Virtual bright-field and dark-field images can be reconstructed by using virtual apertures (Figure 1) applied to a CBED discs resultant from specific grain orientations. From this orientation mapping it becomes clear that neighboring crystallites are not as perfectly aligned with respect to their crystallographic c-axis as previously thought, in line with results from recent polarization-dependent imaging contrast (PIC) mapping using x-ray absorption spectroscopy, albeit for a much lower resolution [3]. Strikingly, we observe structural variations in terms of grain orientation within single crystallites. In order to obtain 3D insight into these structural variations, we additionally prepared a FIB-section from enamel crystallites in a perpendicular direction (sample was cut using FIB with $90^{\circ}$ difference in orientation; see Figure 2). Combined, the use of these virtual apertures highlights that crystal growth directions, present as a record of amelogenesis (enamel formation), vary within single crystallites in three dimensions. This may put emphasis on the role of impurities within the crystallites, which can create locally strained areas within the crystallites. 
Thus, we take advantage of 4D-STEM datasets in counting mode to drastically reduce the acquisition dose while maintaining the structure integrity of enamel crystallites. Valuable information about the structureproperty relationship in this extraordinary biomaterial can be accessed through this technique at room temperature. For example, further analysis of the strain information attained in these datasets can be used to address induced crack deflection in the crystallites and associated toughness mechanisms. [4]
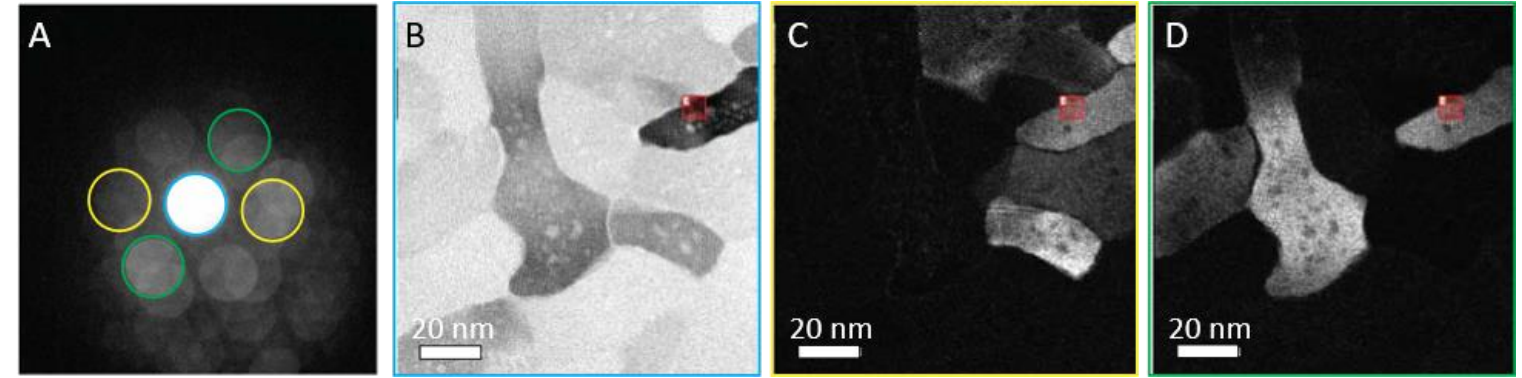

Figure 1. 4D-STEM dataset of beam-sensitive enamel crystallites acquired using K3 IS at 600 fps. A): Convergent beam electron diffraction (CBED) pattern of the red area in (B) within an individual enamel crystallite (images on right). The CBED pattern indicates that this crystallite is close to the [001] zoneaxis. Virtual apertures (colored circles) within the CBED pattern can be used to create B) bright-field (blue) and C,D) dark-field (yellow/green) images with near-atomic resolution over large areas.
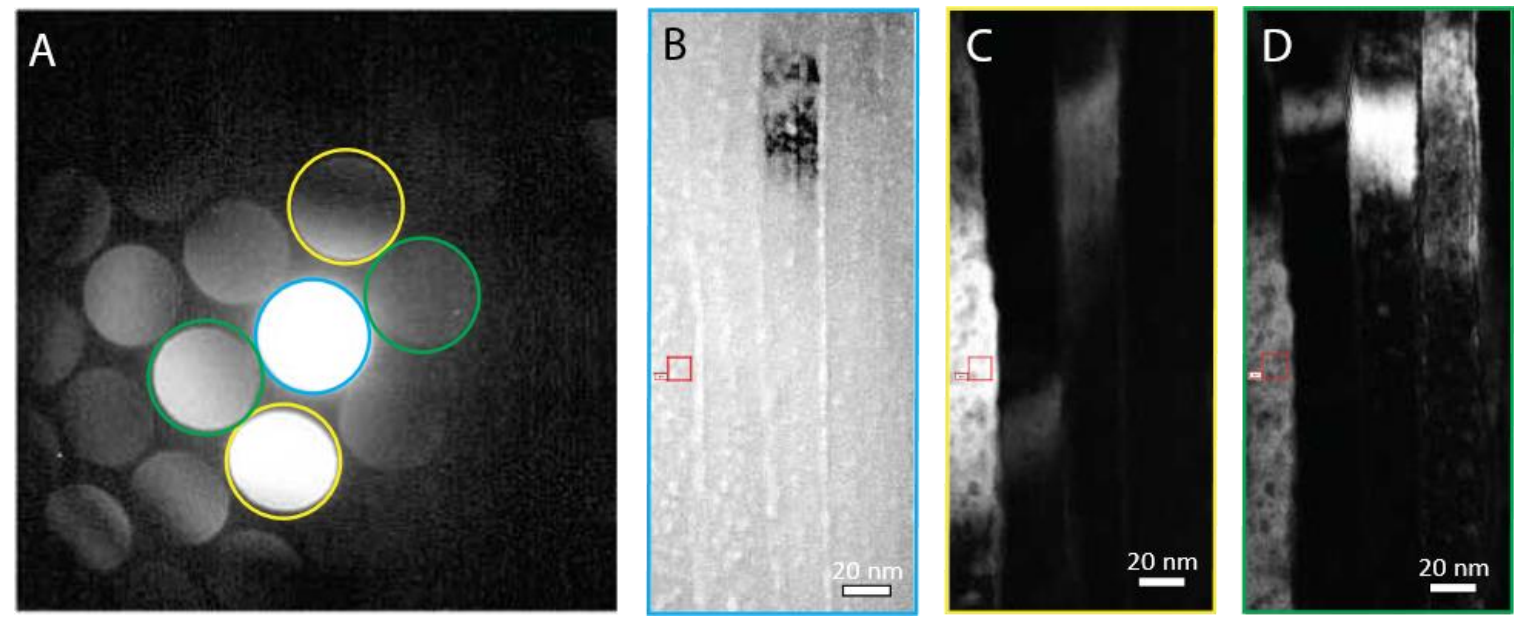

Figure 2. 4D-STEM dataset acquired using K3 IS at $600 \mathrm{fps}$ of enamel crystallites from a FIB-section perpendicular to the section in Figure 1. A): CBED pattern of the red area in (B) within an individual enamel crystallite (images on right). This CBED pattern indicates that the crystallite is along the [1 $1-1$ 1] zone-axis. Virtual apertures (colored circles) within the CBED pattern are used to create B) bright-field (blue) and C,D) dark-field (yellow/green) images.

\section{References}

[1] A. Meldrum, L. M. Wang, and R. C. Ewing, American Mineralogist 1997, 82, 858-869.

[2] Karen A. DeRocher ${ }^{\dagger}$, Paul J.M. Smeets ${ }^{\dagger}$, Berit H. Goodge, Michael J. Zachman, Prasanna V. Balachandrana, Linus Stegbauer, Michael J. Cohen, Lyle M. Gordon, James M. Rondinelli, Lena F. Kourkoutis, and Derk Joester, in review ${ }^{\dagger}$ : contributed equally

[3] Elia Beniash, Cayla A. Stifler, Chang-Yu Sun, Gang Seob Jung, Zhao Qin, Markus J. Buehler \& Pupa U. P. A. Gilbert, Nature Communications, 2019, 10, 4383 
[4] This work made use of the EPIC facility of Northwestern University's NUANCE Center, which has received support from the Soft and Hybrid Nanotechnology Experimental (SHyNE) Resource (NSF ECCS-1542205); the MRSEC program (NSF DMR-1720139) at the Materials Research Center; the International Institute for Nanotechnology (IIN); the Keck Foundation; and the State of Illinois. We acknowledge Gatan Inc (Pleasanton, CA, USA) for the use of the K3 IS camera installed on the JEOL ARM300F at the NUANCE Center. Research reported in this work was supported by the National Institute of Health-National Institute of Dental and Craniofacial Research (NIH-NIDCR) under award number R01 DE025702-01 